\title{
Ink feed control in a web-fed offset printing press
}

\author{
Cristofer Englund • Antanas Verikas
}

Received: 30 May 2007 / Accepted: 8 October 2007 / Published online: 7 November 2007

(C) Springer-Verlag London Limited 2007

\begin{abstract}
Automatic and robust ink feed control in a webfed offset printing press is the objective of this work. To achieve this goal an integrating controller and a multiple neural models-based controller are combined. The neural networks-based printing process models are built and updated automatically without any interaction from the user. The multiple models-based controller is superior to the integrating controller as the process is running in the training region of the models. However, the multiple models-based controller may run into generalisation problems if the process starts operating in a new part of the input space. Such situations are automatically detected and the integrating controller temporary takes over the process control. The developed control configuration has successfully been used to automatically control the ink feed in the web-fed offset printing press according to the target amount of ink. Use of the developed tools led to higher print quality and lower ink and paper waste.
\end{abstract}

Keywords Ink feed control - Colour printing . Multiple models $\cdot$ Integrating controller $\cdot$ Neural networks

C. Englund $(\bowtie) \cdot$ A. Verikas

Intelligent Systems Laboratory, Halmstad University,

Box 823, 30118 Halmstad, Sweden

e-mail: cristofer.englund@ide.hh.se

A. Verikas

e-mail: antanas.verikas@ide.hh.se

\section{A. Verikas}

Department of Applied Electronics,

Kaunas University of Technology,

Studentu 50,

51368 Kaunas, Lithuania

\section{Introduction}

Colour images, as such appearing on a camera display or a computer monitor, are composed of a mixture of three primary colours: red $(\mathrm{R})$, green $(\mathrm{G})$, and blue $(\mathrm{B})$. The $R G B$ primaries correspond to the three types of colour sensing elements-cones-found in the human eye [1]. $R G B$ is an additive colour system which means that the spectra of light coming from the three primary sources are added to reproduce the spectrum of a certain colour. When the three primaries are mixed in equal portions a grey shade is conceived. The lower the intensity the darker the colour appears.

A white substrate, i.e. paper, is usually used in printing. White paper possesses approximately the same reflection coefficient for all wavelengths in the visible spectrum. When the white paper is illuminated, the colour perceived by an observer approximately matches that of the light source. To attain colours during printing, in contrast to the additive system, a subtractive colour system is used where portions of the light are absorbed by the printed ink. The type of ink determines in what part of the spectrum the absorption takes place. The primary colours usually used in four-colour printing are cyan (C), magenta $(\mathrm{M})$, yellow $(\mathrm{Y})$, and black $(\mathrm{K}), C M Y K$. A $C M Y$ overprint creates black colour. However, black ink $(\mathrm{K})$ is also used in printing. Due to economical reasons, black ink often replaces the $C M Y$ overprints. Moreover, black ink is often used to improve the quality of colour pictures. Since colour images are usually obtained in the $R G B$ colour space, while printed using the $C M Y K$ primaries, printing involves the so-called colour separation process, where $R G B$ images are transformed into the $C M Y K$ colour space $[2,3]$.

A printed multi-coloured picture is made of dots of varying size of the four primary colours, CMYK. The left 
side of Fig. 1 shows an example of a colour print. The colour shade perceived by an observer in a small area of a colour print is determined in the halftoning process where dots of varying size (tonal value) are created. The larger the dots, the darker the colour shade. The hue perceived by an observer in a small area of a printed picture is determined by the proportion of the amount of ink of the four primary colours in the area.

After estimation of the dot sizes the dots are transferred onto printing plates in the computer-to-plate (CTP) process. There is a separate plate for each printing colour. The CTP images the dots on thin aluminium plates. The plates are mounted on the plate cylinders of the inking system of the printing press. The right-hand side of Fig. 1 presents an illustration of the inking system. Since both the empty and printable areas on the plate are on the same plane, they are distinguished from each other by some being water receptive and others being ink receptive. During printing, a thin layer of water (dampening solution) is applied to the plate followed by the application of the corresponding ink. The inked picture is transferred from the plate onto the blanket cylinder and then onto the paper.

To achieve the desired colour during printing, the press operator samples the print manually throughout the job run. The sample is compared to the approved sample print and a great effort is made to compensate for colour deviations detected in the print. Since the dot sizes are fixed, the only possibility to change the colour during printing is to change the density of the dots through adjustments of the ink feed into the inking system. Each operator performs the ink feed adjustments based on experience gained from working at that particular press. Typically, the perception of the printed result is very subjective and consequently great variations may appear depending on the operator controlling the process.

By using an automatic control system one can eliminate the inconsistent sampling and subjective colour compensation made by the operator and therefore one can expect a more uniform print quality through the production. Consequently, paper and ink waste will be reduced as the print quality variations decrease. Among the advantages of using an automatic control system are its swiftness, continuous sampling, consistency in control actions, and resistance to wear. Operator's time is also freed up for the benefit of service and maintenance of the printing press equipment.

There have been few successful attempts to automatically control the ink feed in a web-fed offset newspaper printing press. In [4], a knowledge base, build up from observing an experienced operator, is used to help a novice operator to adjust the ink feed to compensate for ink density deviations in the print. The decision support system developed in [5] is used in a wall-covering rotogravure printing industry. The system measures a number of characteristics of the print, including colour. If drift is detected in any of the parameters, the system instructs the operator to make adequate adjustments to the process variables. The system developed in [6] for online ink feed control is able to drive the ink density of the print to the desired target density level.

In all the aforementioned works, the ink feed control is based on controlling the ink density measured on a solid print area, as that shown at the top of Fig. 2. However, printed pictures are made of dots. Since not only the ink density, but also the size of the dots may vary in the printing process, ink density does not provide enough information for controlling the printing process. The amount of ink, integrating both the ink density and the dot size, should be used instead. Therefore, we control the printed amount of ink estimated in the double grey bar, shown in the bottom picture of Fig. 2. The double grey bar consists of two parts, one part is printed using the black ink and the other part using the cyan, magenta, and yellow inks.

\subsection{Assessing the amount of ink}

Print quality is a wide notion in the printing industry. Colour deviation, colour registration, dot size, and dot shape are common parameters that characterise print quality. In this work, print quality is defined as the disparity (distance) between the colour of the sample and the reference print. We use the approximately uniform $L^{*} a^{*} b^{*}$ colour space [7] to evaluate the disparity. Therefore, the $R G B$ image recorded
Fig. 1 Left: An example of dots creating a colour print. Right: The inking system
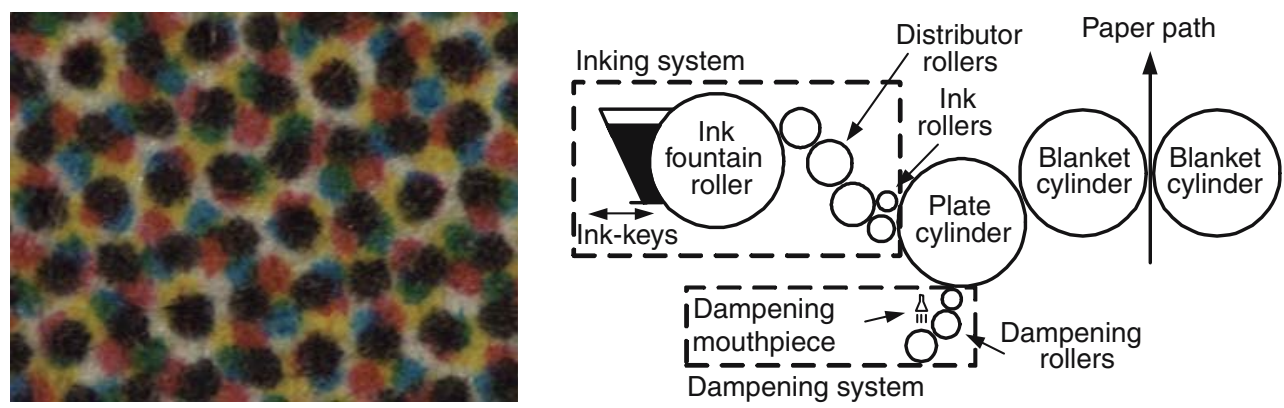


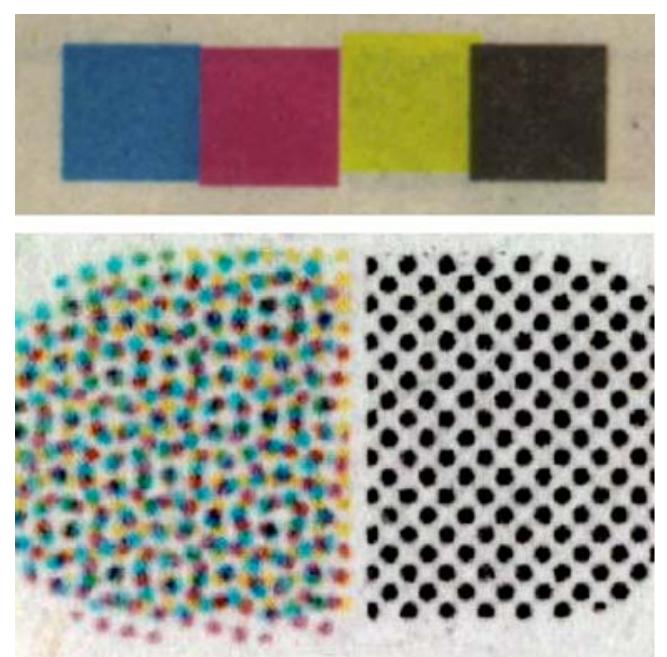

Fig. 2 Top: Full tone target areas. Bottom: The double grey bar

from the double grey bar by a colour $C C D$ camera is transformed into the $L^{*} a^{*} b^{*}$ counterpart. The $L^{*} a^{*} b^{*}$ values are, however, not easily assessable by the press operator who usually wants to know the deviation of the amount of cyan, magenta, yellow, and black inks from the required levels. We use the technique proposed in [8] to estimate the amount of ink in the double grey bar from the $L^{*} a^{*} b^{*}$ components. The neural networks-based technique transforms the pair of $L^{*} a^{*} b^{*}$ values recorded from the two parts of the double grey bar into the amount of $C, M, Y$, and $K$ inks. The neural network is trained using colour patches printed with the constant ink density and varying tonal value (the percentage of area covered by the ink) to predict the amount of ink printed on the paper. If the ink density used to print a test patch is equal to that kept when printing patches for neural network training, the printed amount of ink estimated in the test patch may vary between 0 and 100 . If the ink density of the test patch exceeds the one used to print the training patches, the measured amount of ink may exceed 100 (for an area with 100\% ink coverage). In this work, a given amount of ink (estimated in the way discussed above) is the target signal the controller has to maintain.

\section{Printing process variables and solution approach}

The printing press operator samples the print throughout the job run. As colour deviation from the approved sample print is detected, the ink feed is either increased or decreased by adjusting the ink keys. The ink keys are situated at the bottom of the ink tray (Fig. 1, right). For the press considered here, there are 36 ink keys for each colour and side of the web. The ink key adjusts the ink feed in an approximately $4 \mathrm{~cm}$ wide ink zone (see Fig. 3). Model- based control systems are common in industry since process models have the ability to mimic complex direct and inverse process behaviour. Multiple models-based controllers have been shown to be efficient in many industrial control applications due to their ability to improve stability and increase the modelling performance [9-11].

We have recently proposed an approach to automatic data mining and neural networks-based printing press modelling [12]. Based on this approach we have developed a multiple models-based technique for ink feed control, which has shown good performance in controlling the ink feed in a web-fed offset printing press [13]. There are a number of models of different complexity, specialised and general ones, engaged in controlling the printing process. The specialised models are trained on specialised data sets, while the general models are trained on the union of the data sets used to train the specialised models. A committee of specialised models is also incorporated into the set of multiple models. The models are built from historical process data and are automatically updated when such a need is detected. The data collected in one ink zone are called specialised data and thus are used to train the specialised models. Two types of models are built: inverse models, where the ink key opening value constitutes the model output, and direct models, where the printed amount of ink constitutes the output. Depending on the modelling task, inverse or direct, different combinations of process parameters are utilised.The process parameters used to model one ink $(C, M, Y$, or $K)$ are given as:

$x_{1} \quad$ Printing speed in copies per hour.

$x_{2} \quad$ Ink fountain roller speed.

$x_{3} \quad$ Ink temperature. The temperature of the ink in the ink tray. The temperature affects the viscosity of the ink such that the higher the temperature the

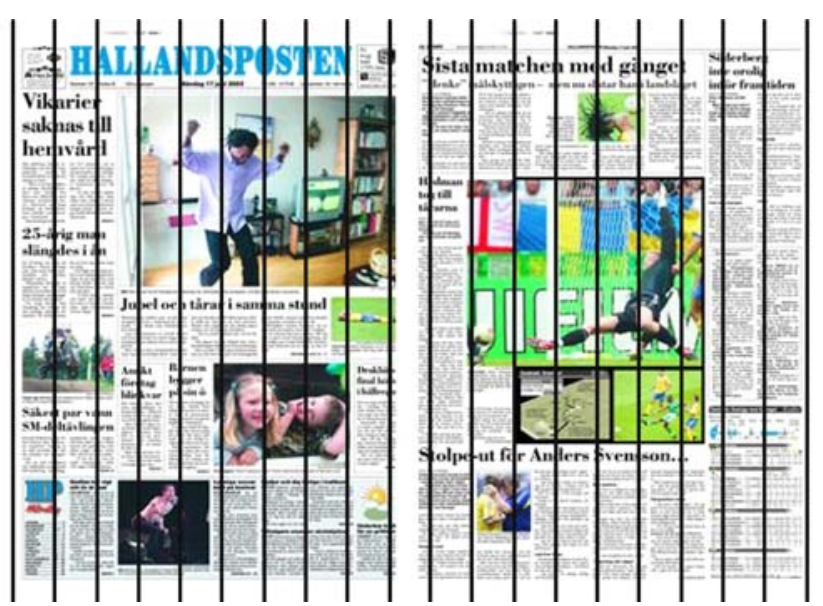

Fig. 3 An example of how the ink zones subdivide the paper fold 
lower the viscosity and thus the ink flows through the inking system easier.

$x_{4,5,6}$ Estimated ink demand for the current, adjacent to the left, and adjacent to the right ink zones, respectively. The ink demand equals the percentage of area covered by ink in the corresponding ink zone.

$x_{7,8,9}$ Ink key opening for the current, adjacent to the left, and adjacent to the right ink zones, respectively.

The signal controlling the amount of ink dispersed on the paper.

$x_{10} \quad$ Amount of ink of a specific colour estimated from the double grey bar.

In direct modelling, $x_{10}(t+1)$ is the model output. However, for inverse modelling, where the modelling task is to predict the ink key opening, the $x_{10}(t+1)$ value is used as an input parameter, while the parameter $x_{7}(t+1)$ constitutes the model output. The variables $x_{7}$ and $x_{10}$ are used from both the current $(t)$ and the next $(t+1)$ time step. Experimental studies have shown that no further performance gain is achieved by exploiting more previous time steps, e.g. $(t-1)$ or $(t-2)$. The variables $x_{4,5,6}$ describe the ink demand in the current $\left(x_{4}\right)$ and the two adjacent ink zones $\left(x_{5}, x_{6}\right)$. Since ink flows between adjacent zones in the printing press, the variables $x_{4,5,6}$ are replaced by their mean, $\overline{x_{4,5,6}}$, in the models. For simplicity, we denote the variables incorporated in the direct and inverse model as:

$v^{d}=\left[x_{1}(t), x_{2}(t), x_{3}(t), \overline{x_{4,5,6}(t)}, x_{7}(t+1), x_{7}(t), x_{8}(t), x_{9}(t), x_{10}(t)\right]$ $v^{i}=\left[x_{1}(t), x_{2}(t), x_{3}(t), \overline{x_{4,5,6}(t)}, x_{7}(t), x_{8}(t), x_{9}(t), x_{10}(t+1), x_{10}(t)\right]$

It should be noted that these variables are used to train the models. When the models are used for control, the variable $x_{7}(t+1)$ is replaced by the output of the inverse model $u(t+1)$, and $x_{10}(t+1)$ is replaced by the desired amount of ink $y^{\text {des }}$. Note also that the ink key opening value varies in the range $[0,100]$. To obtain data necessary for modelling, a web-fed offset newspaper printing press was equipped with an online press monitoring system. A detailed description of the monitoring system can be found in [13].

An artificial neural networks-based modelling approach has been adopted in this work. Artificial neural networks proved themselves to be powerful tools in process and system modelling. However, like other nonlinear models neural networks-based models may run into generalisation problems when data outside the training region need to be processed. Such situations are encountered in the printing industry, since new unexpected jobs may always appear. To cope with the problem, we complement the multiple models-based control configuration with the integrating controller in this work. Integrating controllers are known for their simplicity. Typically, they have only one parameter and use only the error signal to estimate the new control signal. To be able to control the process at all times, we suggest building a hybrid control system consisting of an integrating controller and a multiple models-based controller.

\section{Methods}

\subsection{Printing process modelling}

Due to wear of the printing press, the process can be classified as slowly time-varying. In addition, depending on a printing job, the time the process stays in a predefined part of the input variable space may vary significantly, from minutes to several days. If the process starts to operate in a new region of the input variable space, different from the training region, the model performance may deteriorate significantly. To handle such situations, we have recently proposed an adaptive data mining and modelling approach [12]. The data mining tool monitors the process data and keeps an up-to-date data set of a reasonable size characterising the process. The adaptive modelling is aimed at building models of optimal complexity. Starting with a linear model, a number of nonlinear models of increasing complexity (MLP with an increasing number of hidden units) are built; then, a model with the lowest generalisation error is selected for modelling the process. During the process run, the need to update the models is automatically detected and the models are retrained. In this work, we use this technique to create and update the process models.

Four types of models are used in this work for modelling the printing process:

- A model specific for each ink key/zone. These models are called specialised, since they have specific knowledge about a certain ink key/zone. Each specialised model is trained using data from a specific ink zone.

- A committee of specialised models. Specialised models implementing similar functions are aggregated into a committee. In [14] we developed an approach for building committees of models where both the number of committee members and the aggregation weights of the members are data dependent. We use this approach to create committees of models.

- A nonlinear general model that is built using the data from all the ink-zones. The general model is built using more data than the specialised one and therefore it generalises better than the specialised model.

- A linear general model built using data from all the ink zones. 
The specialised models and committees of the models provide the highest modelling accuracy. However, due to the limited training data set used, the models may run into generalisation problems. In such situations, general models are used instead, which are built using much more data points than the specialised ones. Since the complexity of the models is determined automatically, the general model may be linear or nonlinear. If a nonlinear general model is automatically selected, a linear general model is also built. The linear general model exhibits the lowest modelling accuracy but the best generalisation ability.

\subsection{Ink key control}

In the control configuration developed, the sampling time is approximately $100 \mathrm{~seconds}$, i.e. the time needed to traverse the camera once over the paper web, to take an image of each of the 36 double grey bars and then return to the initial position.

\subsubsection{Multiple models-based controller design}

A detailed description of the multiple models-based design that we previously developed for ink feed control can be found in [13]. Here we provide only a brief summary of the technique.

Figure 4 illustrates the multiple models-based configuration, where the denotation IM stands for inverse model and DM means direct model. Models incorporated in the control configuration are:
Sing a single specialised model
Com a committee of specialised models
LGen a single general linear model
NLGen a single general nonlinear model

The control configuration functions as follows. The control signal $u$ is given by the output of one of the inverse models. We assume that the inverse model output is normally distributed with the mean given by the model output and the standard deviation $\sigma$. A large standard deviation of the predicted control signal indicates model uncertainty.

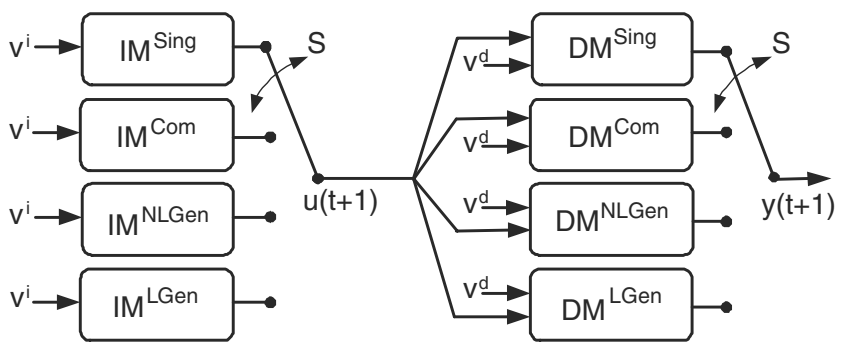

Fig. 4 The multiple models-based control configuration
By sampling from the distribution of the inverse model output, as suggested in [15], we produce a set of control samples $U=\left[u_{1}, u_{2}, \ldots, u_{D}\right]$ that are evaluated using the direct model (see Fig. 4). The number of samples $D$ is determined by the model standard deviation $\sigma$. The larger the $\sigma$ the more samples are generated.

The outputs of the inverse model $u$ and the direct model $y$ are given by

$u(t+1)=f^{i}\left(v^{i} ; \theta^{i}\right)$

$y(t+1)=f^{d}\left(v^{d} ; \theta^{d}\right)$

where $\boldsymbol{\theta}$ is the model parameter vector and the $f$ functions can be either linear or nonlinear.

The control signals $u_{i 1}, u_{i 2}, \ldots, u_{i D}$ generated by each of the inverse models $(i=1, \ldots, 4)$ are used to calculate the outputs of the direct models $y_{11}, y_{21}, \ldots y_{41}, \ldots, y_{4 D}$. The output $y_{i j}$ is given by

$y_{i j}(t+1)=f_{i}^{d}\left(v_{i j}^{d} ; \theta^{d}\right)$

where, $i=1, \ldots, 4$ refers to a model. The model selected is that which minimises the error $e_{i j}$, the difference between the output of the direct model $y_{\mathrm{ij}}(t+1)$ and the target (the desired amount of ink) $y^{\text {des }}: e_{i j}=\left\|y_{i j}(t+1)-y^{\text {des }}\right\|$. Having found all $e_{i j}$, the indices $p, q$ of the control signal $u_{p q}$ sent to the press are found as follows:

$p, q=\arg \min _{i, j} e_{i j}$

The control signal selected, $u_{p q}$, is denoted as $u^{m m}(t+1)$. If for a given $\mathbf{v}, e_{p q}>\beta$ and $p \neq 3$, the linear general model is used to avoid using the nonlinear model with a large prediction error.

\subsubsection{Robust ink feed control}

Figure 5 illustrates the case where the neural network-based controller comes into generalisation problems. The left graph shows the ink key control signal (above) and the measured amount of ink along with the target amount of ink indicated by the solid line (below). Initially the multiple models-based controller runs the process. At sample numbers 7 and 9, the target amount of ink is changed. Accordingly, the multiple models-based controller is adjusting the ink key opening to obtain the desired amount of ink. As can be seen, the ink key adjustments do not bring the process output to the desired level. At sample numbers 21 and 22 , the control action from the multiple models-based controller is manually overridden and the desired target level is reached. The right side of Fig. 5 shows the origin of the problem. 
Fig. 5 Left (top): Ink key opening and (bottom): the measured and the target (solid line) amount of ink. Right: The input data projected onto the space spanned by the first two principal components
Black ink key opening

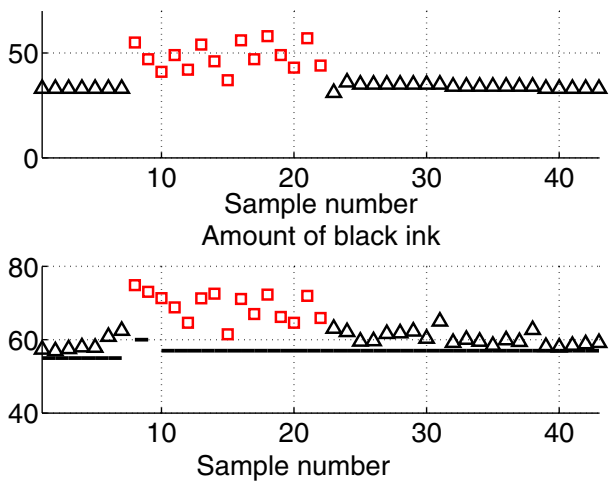

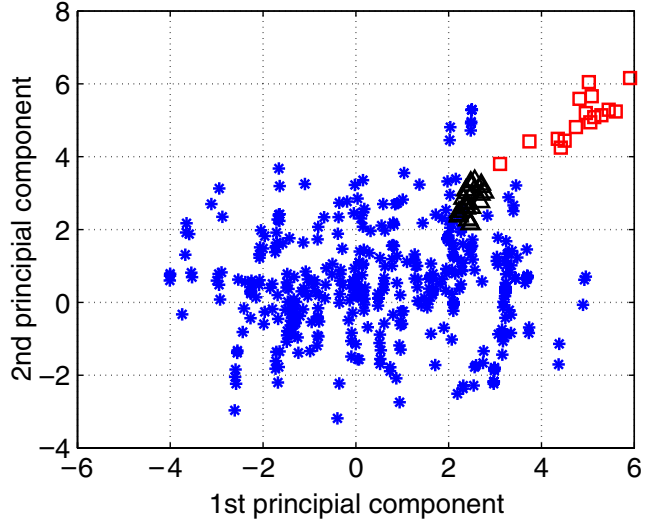

The right side of Fig. 5 shows the training data, indicated by the ${ }^{*}$, and the data from the current job, indicated by the $\triangle$ and $\square$, projected onto the first two principal components of the training data. We clearly see that the data indicated by the $\square$ are well separated from the training data. It is obvious that one can expect the multiple models-based controller to succeed only if the models are retrained. However, data are to be collected for retraining the models. During this period we suggest using an integrating controller. Though with lower accuracy, the integrating controller can handle the process temporarily.

We suggest using the difference between the predicted amount of ink $y$ and the measured amount of ink at time $t$, $y^{\text {mes }}(t)$ to detect these situations. The schematic illustration of the robust ink feed controller is shown in Fig. 6. The control signal $u(t+1)$ estimated by the controller is determined as:

$u(t+1)= \begin{cases}u^{m m}(t+1) & \text { if }\left\|y^{m e s}(t)-y(t)\right\|<\xi \\ u^{i c}(t+1) & \text { otherwise }\end{cases}$

where $u^{m m}(t+1)$ is the ink key opening predicted by the multiple models-based controller and $u^{i c}(t+1)$ is the ink key opening predicted by the integrating controller. By using this approach the process is controlled either by the integrating or multiple models-based controller.

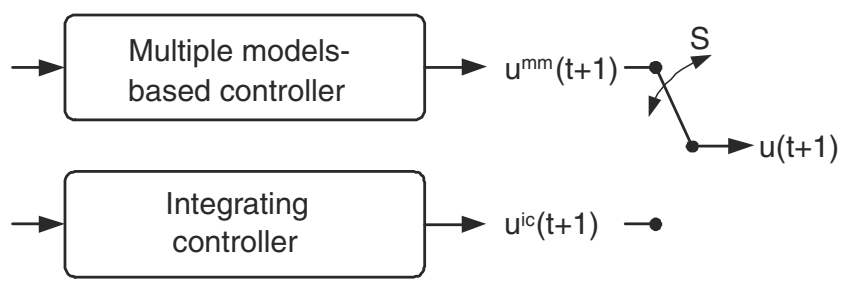

Fig. 6 The proposed control configuration

\subsubsection{Integrating controller design}

The control signal generated by the integrating controller is estimated as

$u^{i c}(t+1)=u(t)+K\left(y^{\text {des }}-y^{\text {mes }}(t)\right)$

where $u(t)$ is the ink key opening at the time step $t, K$ is the integrating factor, $y^{d e s}$ is the desired amount of ink, and $y^{\text {mes }}(t)$ is the measured amount of ink at the current time step $t$. It should be noted that the ink key opening is a discrete signal bounded in the range $[0,100]$.

\section{Experimental investigations}

The experiments were conducted during normal production at the printing shop. The goal of the experiments was to investigate three matters:

1. To find the appropriate value of the parameter $K$ for the integrating controller

2. To compare the two control configurations

3. To demonstrate the benefit of the robust control system

\subsection{Selecting the parameter $K$}

To find the appropriate $K$ value, the parameter was varied between 0.2 and 2.5. In Fig. 7, we present three examples of control and output signals for different $K$ parameter values. The top graphs show the control signal while the lower graphs present the measured and the desired (solid line) amount of ink. The desired amount of ink is constant during the experiment. The controller starts running the process at sample 4, where the solid line appears. As can be seen, the larger the $K$, the larger is the control action.

It was found that $K=0.7$ is a good choice since at this value, on average, the controller was reasonably fast and not too sensitive to noise. As can be seen in Fig. 7, at 

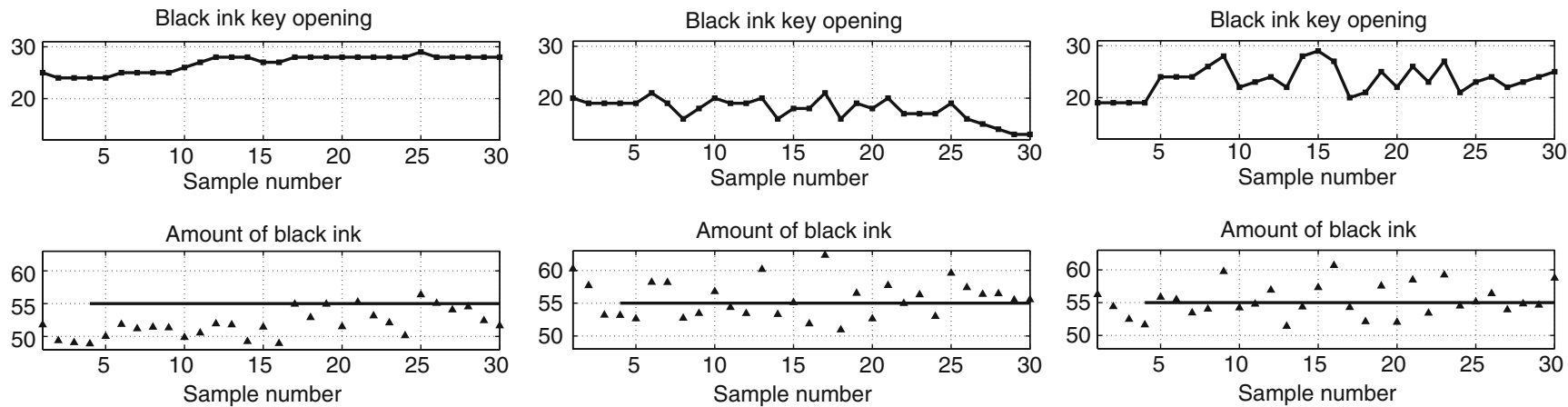

Fig. 7 The control signal (top) and the measured amount of ink along with the desired amount (solid line) of ink for different $K$ values (bottom). $K=0.2,0.7$, and 1.4 for the left, middle, and right graphs, respectively

$K=0.2$ the rise time is very long. At $K=1.4$ both the control signal and the output signal are rather noisy.

\subsection{Comparing the controllers}

The controllers have been used in the same ink zone for the same printing job. We begin by saving the initial settings for the press and start the experiment using one of the controllers. Then we restore the settings of the printing press and continue the same experiment with the other controller. Two issues were studied: the rise time and the sensitivity to noise.

\subsubsection{Rise time}

A short rise time is desirable to reduce paper waste. In Fig. 8, we present the response from the two controllers operating on the same ink key for two different colours. For each colour, the left graphs show the results from the integrating controller whereas the right graphs present the results from the multiple models-based controller. The top graphs show the ink key control signal and the bottom graphs present the measured and the target (solid line) amount of ink. The controller is used from sample number 3 , where the solid line appears. In the figures, ID stands for ink demand.

As can be seen, the integrating controller requires more samples to drive the output to the desired target level. The multiple models-based controller has a shorter rise time than the integrating controller.

Figure 9 presents two more control examples. The results presented are for the case where the target amount of ink is less than the initial printed amount of ink. Again, for both examples, the multiple models-based controller drives the amount of ink to the desired level faster than the integrating controller.

One can make the integrating controller faster by increasing the value of $K$. However, larger values of $K$

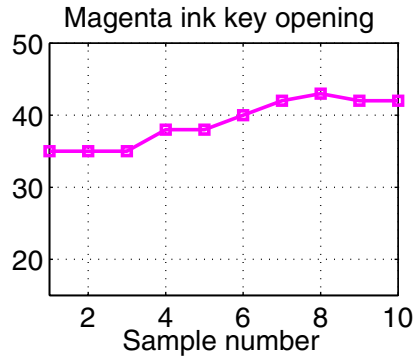

Amount of magenta ink, ID: $38 \%$

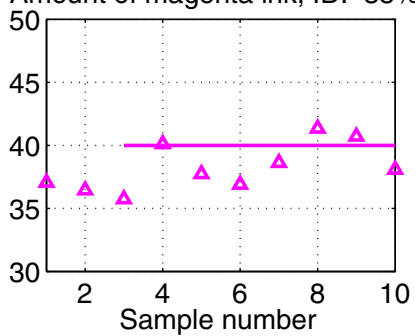

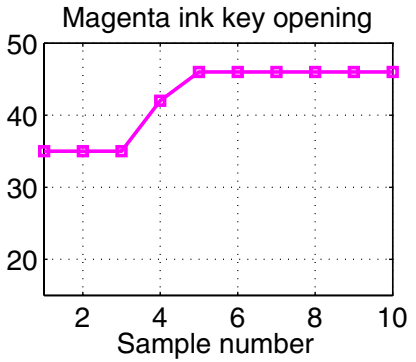

Amount of magenta ink, ID: $38 \%$

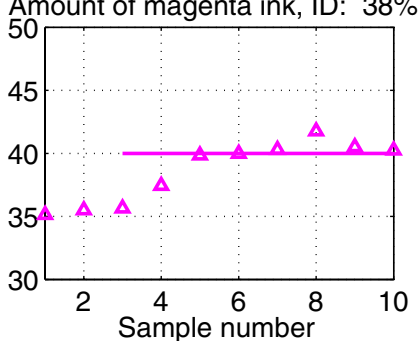

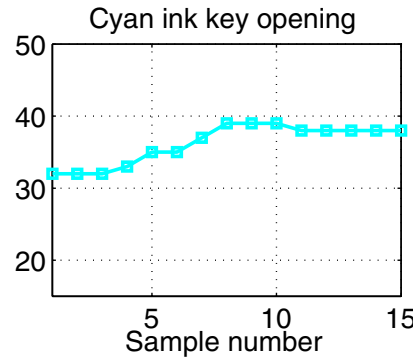

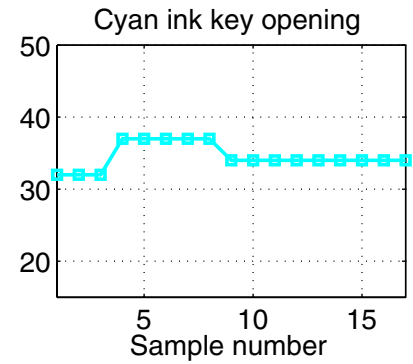

Amount of cyan ink, ID: $25 \%$

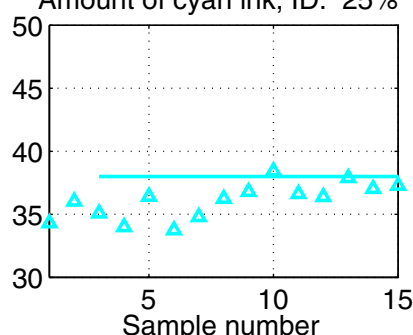

Amount of cyan ink, ID: $25 \%$

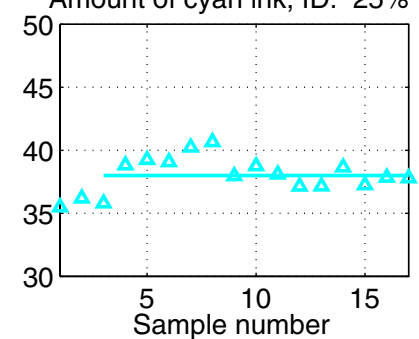

Fig. 8 Results from the integrating controller (first and third columns) and the multiple models-based controller (second and fourth columns) 


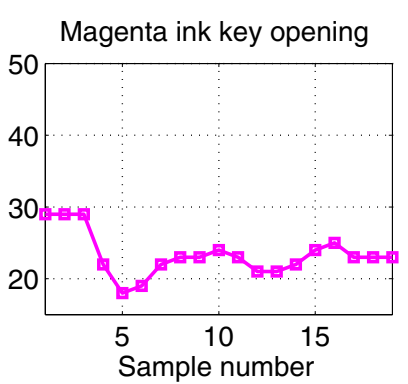

Amount of magenta ink, ID: $29 \%$

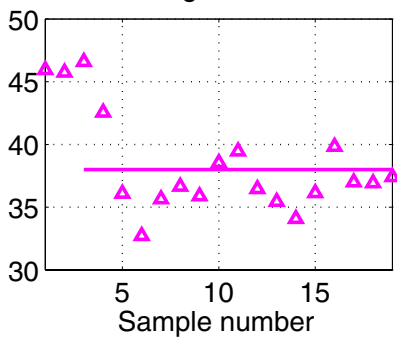

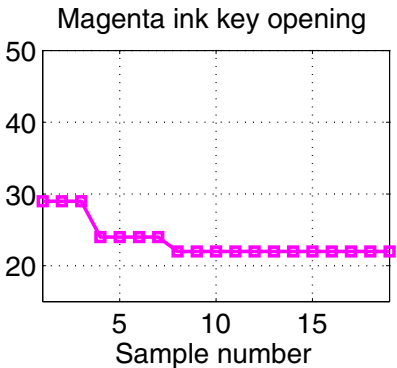

Amount of magenta ink, ID: $29 \%$

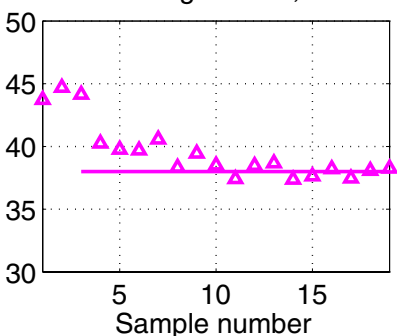

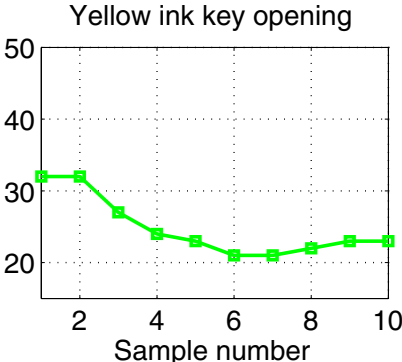

Amount of yellow ink, ID: $29 \%$

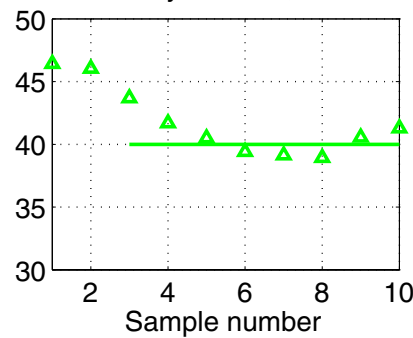

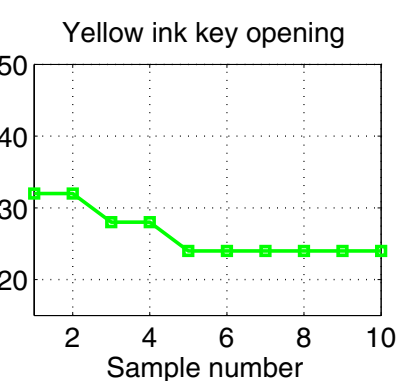

Amount of yellow ink, ID: $29 \%$

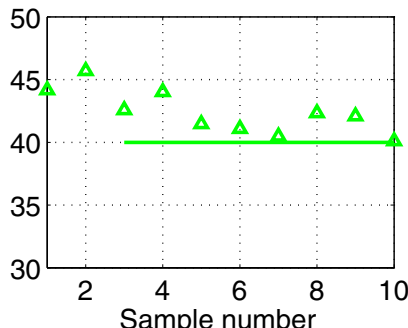

Fig. 9 Results from the integrating controller (first and third columns) and the multiple models-based controller (second and fourth columns)

make the process output too noisy. In fact, an adaptive value of $K$ is required. Analysis of the inverse model output presented below illustrates the adaptivity of the ink key adjustment step size obtained from the inverse model. In Figs. 10 and 11, we present the inverse process model simulation results for the cyan colour. During the simulations the ink temperature, the printing speed, the ink fountain roller speed, and the target amount of ink (set to 42) were held constant. The current amount of ink was set to 34 and 50 for the simulations presented on the left and right sides of the figures, respectively. The ink temperature was set to $24^{\circ} \mathrm{C}$ and $23^{\circ} \mathrm{C}$ for the simulations presented in Figs. 10 and 11 , respectively.

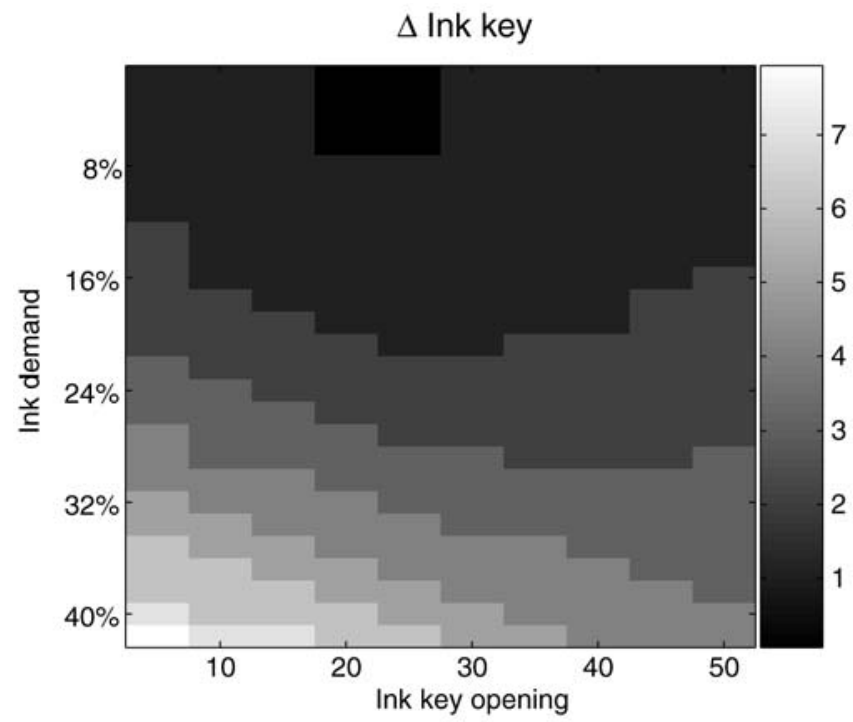

The graphs presented in Figs. 10 and 11 illustrate the predicted ink key opening adjustment ( $\Delta$ ink key) needed to drive the current amount of ink to the target level as a function of the ink demand and the current ink key opening. The grey level in the images indicates the magnitude of the ink key change. We clearly see that the magnitude is different for different states of the process, i.e. for different current ink key openings and different ink demands. As can be seen from Figs. 10 and 11, the magnitude of the ink key change is larger for the lower temperature, as expected. Hence, the simulation results illustrate the adaptivity of the ink key adjustment available from the inverse model. The integrating controller, however, calculates the ink key

Fig. 10 The $\Delta$ ink key opening needed to drive the current amount of ink to the target level as a function of the ink demand and the current ink key opening. The ink temperature is $24^{\circ} \mathrm{C}$. The current amount of ink is equal to 34 (left) and 50 (right) 

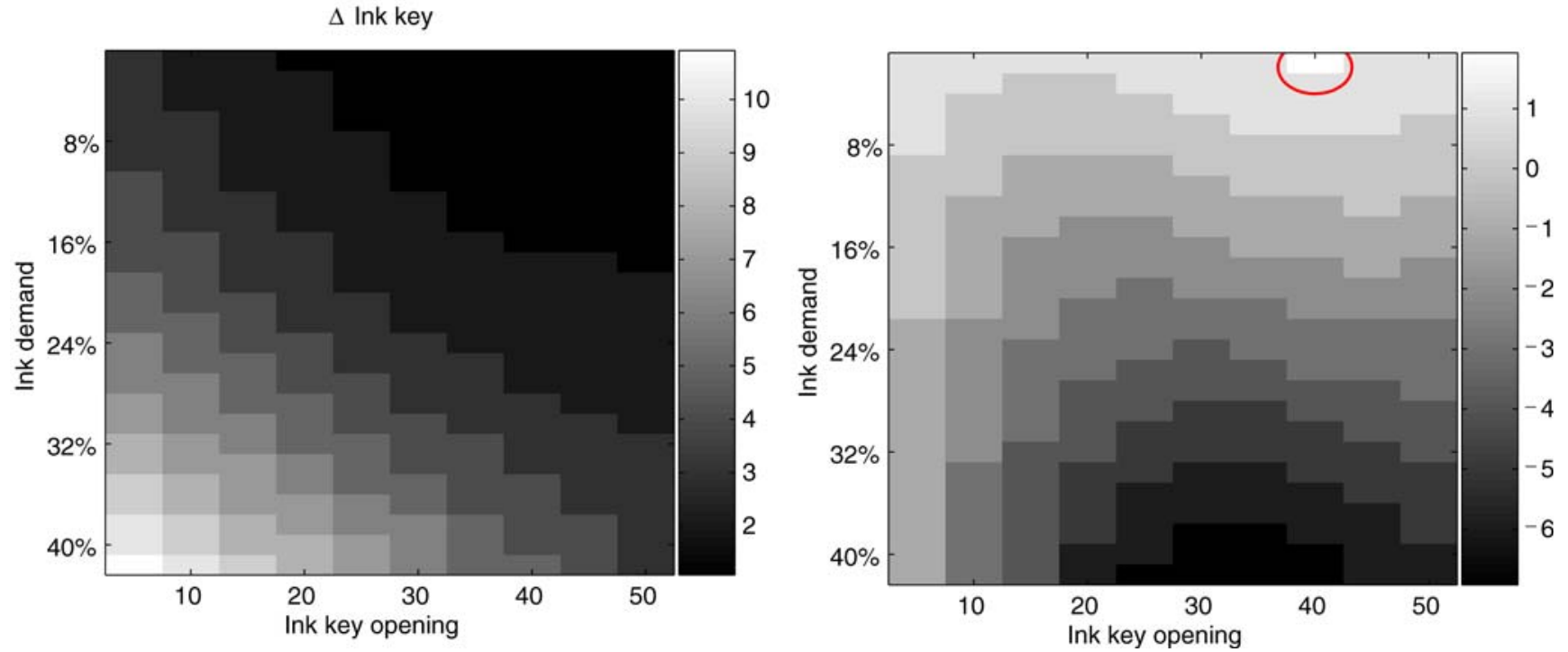

Fig. 11 The $\Delta$ ink key opening needed to drive the current amount of ink to the target level as a function of the ink demand and the current ink key opening. The ink temperature is $23^{\circ} \mathrm{C}$. The current amount of ink is equal to 34 (left) and 50 (right)

adjustment signal only with respect to the current error of the amount of ink. In Figs. 10 and 11, examples of clearly erroneous predictions are encircled (for example, positive $\Delta$ ink key value despite the fact that the current amount of ink is larger than the target).

Figure 12 illustrates the uncertainty of the model predictions (in relative units) shown on the right sides of Figs. 10 and 11. As can be seen, the model is rather uncertain about the erroneous predictions. It should be kept in mind that, in this work, the problem of uncertain predictions is investigated by exploring a large number of control signals.

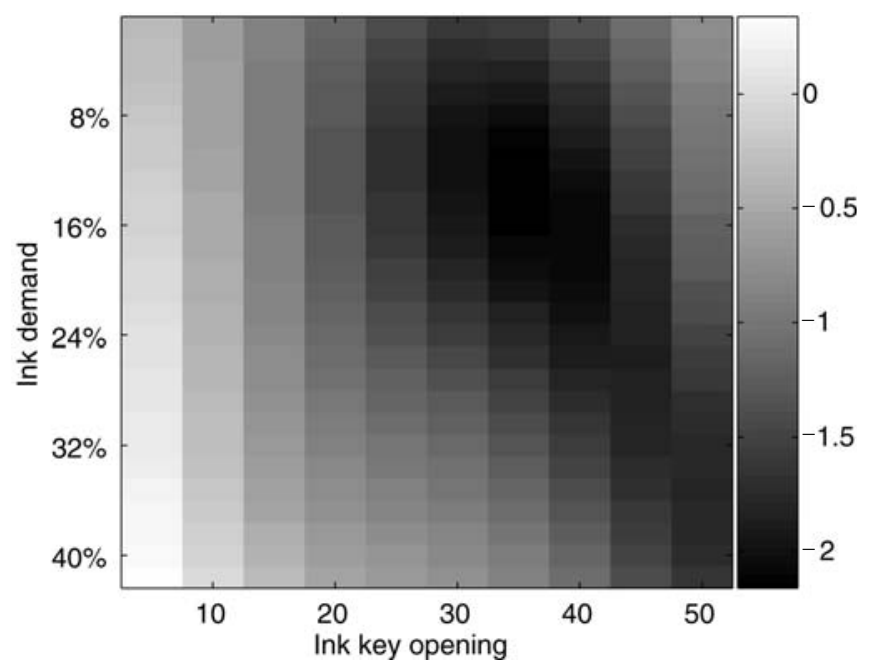

\subsubsection{Noise in the control and output signal}

It is desirable to produce prints with as low colour variations between different copies as possible. Our previous studies have shown that, on average, the noise level $\sigma$ in the measured amount of ink is approximately 2 units [12]. On average, larger root mean square error (RMSE) values were observed for the integrating controller than for the multiple models-based controller. Table 1 summarises the RMSE of the output signal for the examples presented in Figs. 8 and 9. The superiority of the multiple models-based controller should be obvious.

Fig. 12 The uncertainty of the predictions shown at the right side of Figs. 10 (left) and 11 (right)

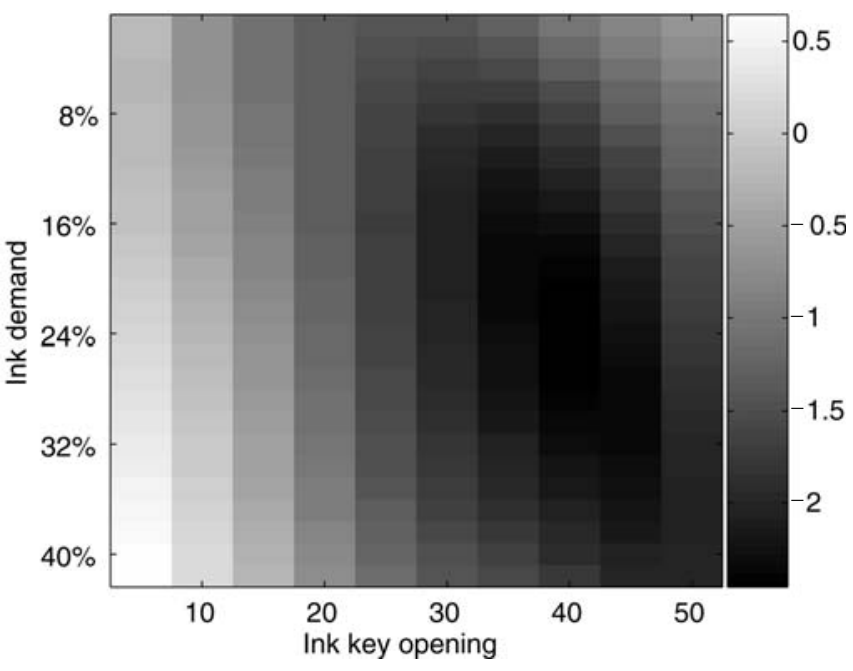


Table 1 The RMSE of the measured amount of ink for the experiments illustrated in Figs. 8 and 9, where IC stands for the integrating controller, $\mathrm{MM}$ for the multiple models-based controller and $\mathrm{C}, \mathrm{M}$, and $\mathrm{Y}$ stand for a corresponding colour

\begin{tabular}{lllll}
\hline Controller & Fig. 8 (M) & Fig. 8 (C) & Fig. 9 (M) & Fig. 9 (Y) \\
\hline IC & 1.68 & 3.79 & 3.70 & 2.91 \\
MM & 1.12 & 1.97 & 1.65 & 1.94 \\
\hline
\end{tabular}

Results obtained from long-term experiments are discussed below.

\subsection{Robust ink feed control}

The printing process may start to operate in a new region of the input variable space, different from the training region, as was discussed previously and is illustrated in Fig. 5. Therefore, we use the robust approach to ink feed control, where the integrating controller and the multiple modelsbased controller are combined. Figure 13 presents an example illustrating the benefit of the approach proposed. The top graph at the left of Fig. 13 shows the ink key control signal. The target, indicated by the solid line, and the measured amount of ink $(\triangle, \diamond)$ are shown in the middle graph. The model prediction error and the threshold of the error are presented in the bottom graph. The image presented on the right side of Fig. 13 shows the input data projected onto the first two principal components computed using the training data. We distinguish three regions in the control sequence. At the beginning, the multiple modelsbased controller runs the process. At the point where the prediction error of the amount of ink exceeds the threshold, $\xi=6$, the integrating controller takes over the control (samples indicated by $\diamond$ ). The integrating controller brings the process to the target amount of ink and the prediction error of the model is low again. As can be seen from

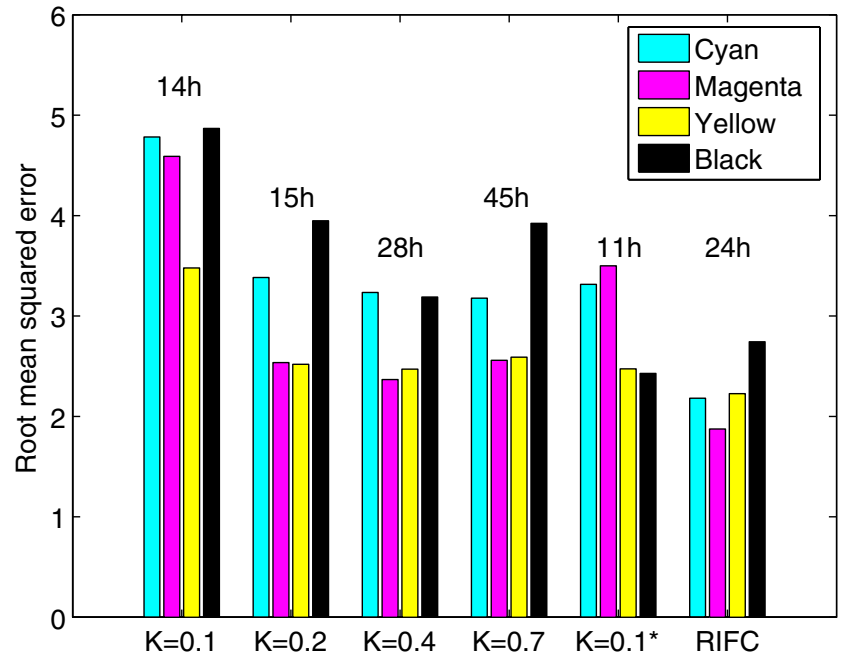

Fig. 14 Long-term experiment results for the integrating controllers and the robust ink feed controller. The $*$ indicates the model with adaptive gain

Fig. 13, the data resulting in the high prediction error, indicated by $\diamond$, appear at the edge of the main bulk of the training data, indicated by *. This explains why the multiple models-based controller has problems with these data points.

\subsection{Long-term experiments}

To demonstrate the benefit of the control configuration proposed we carried out long-term experiments for a number of different integrating controllers and the proposed robust ink feed controller (RIFC). Integrating controllers with $K=0.1,0.2,0.4,0.7$, and adaptive $K$ given by:

$K=\left\{\begin{array}{l}1 \text { if }\left\|y^{\text {mes }}(t)-y(t)\right\|>3 \sigma \\ 0.1 \text { otherwise }\end{array}\right.$
Fig. 13 Left top: The ink key opening. Left middle: The measured and the target (solid line) amount of ink. Left bottom: The error between the predicted and measured amount of ink and the threshold of the error (dashed line). Right: The data projected onto the first two principal components computed using the training data
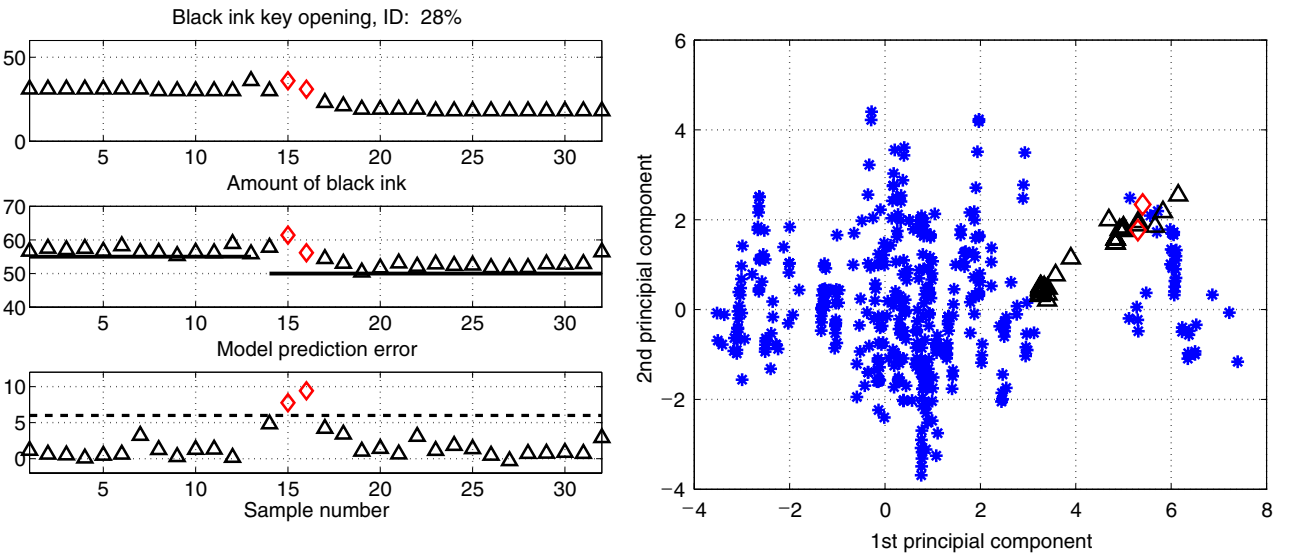

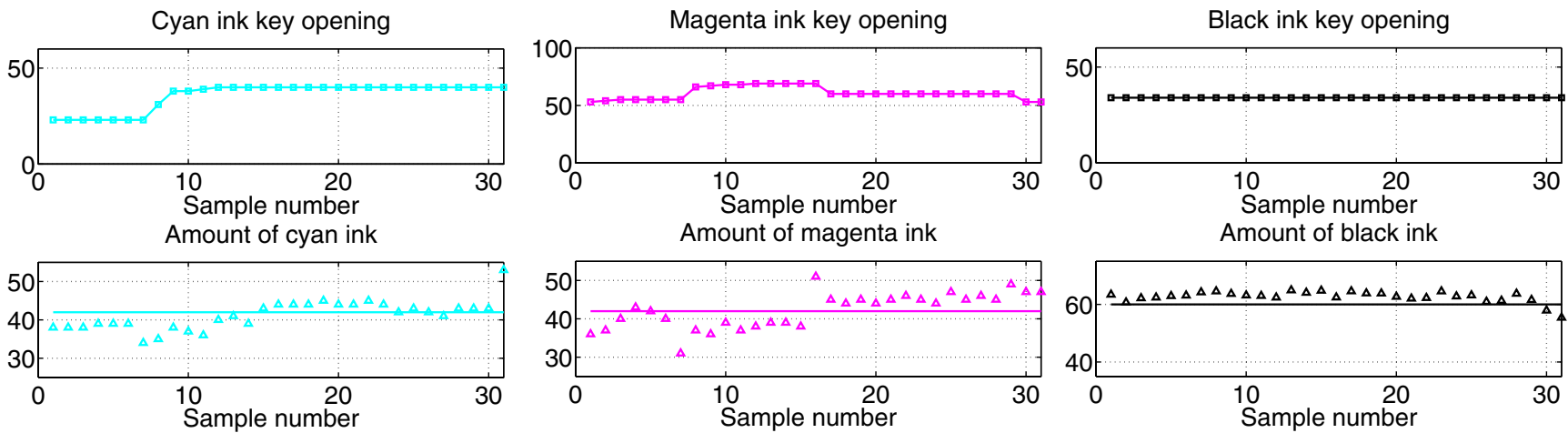

Fig. 15 The control signal (top) and the measured signal along with the desired (solid line) amount of ink (bottom) obtained from the integrating controller with adaptive gain

have been used in the experiment. The integrating controller with adaptive gain was implemented to lower the rise time and the standard deviation of the process output. Figure 14 summarises the results of the experiments via the average RMSE of the process output.

In Fig. $14, K=0.1^{*}$, indicates the integrating controller with adaptive gain. During the long-term experiment the controllers were used for several products and different ink zones. The time the controllers have been used is displayed above the error bars.

As can be seen from Fig. 14, the RIFC provided the best performance, and even small reductions in the process output error result in a reduction in paper waste.

In Figs. 15 and 16 we explore several "control curves" obtained from the integrating controller and the RIFC, respectively. As can be seen, the integrating controller has problems removing stationary errors, since the gain is too small $(K=0.1)$. However, if the gain is increased, the output signal becomes noisy. The robust ink feed controller manages, however, to keep the output well around the target amount of ink.

\section{Conclusions and discussion}

A robust technique for ink feed control in a web-fed offset printing press was presented. The technique combines an integrating controller and a neural multiple models-based controller. We have shown during long-term experiments that the multiple models-based controller is superior to the integrating controller by both a lower rise time and lower noise in the output signal. However, as the process starts to operate in a new part of the input space, the multiple neural models may run into generalisation problems. Such situations are automatically detected and the integrating controller temporarily takes over the control of the process. We have shown experimentally that the proposed technique is able to automatically control the ink feed in the newspaper printing press according to the target amount of ink. Although the improvement obtained from the RIFC is only about $20 \%$, if compared to the integrating controller with the adaptive gain this can, in the long run, bring in large savings in the form of reduced paper and ink waste.
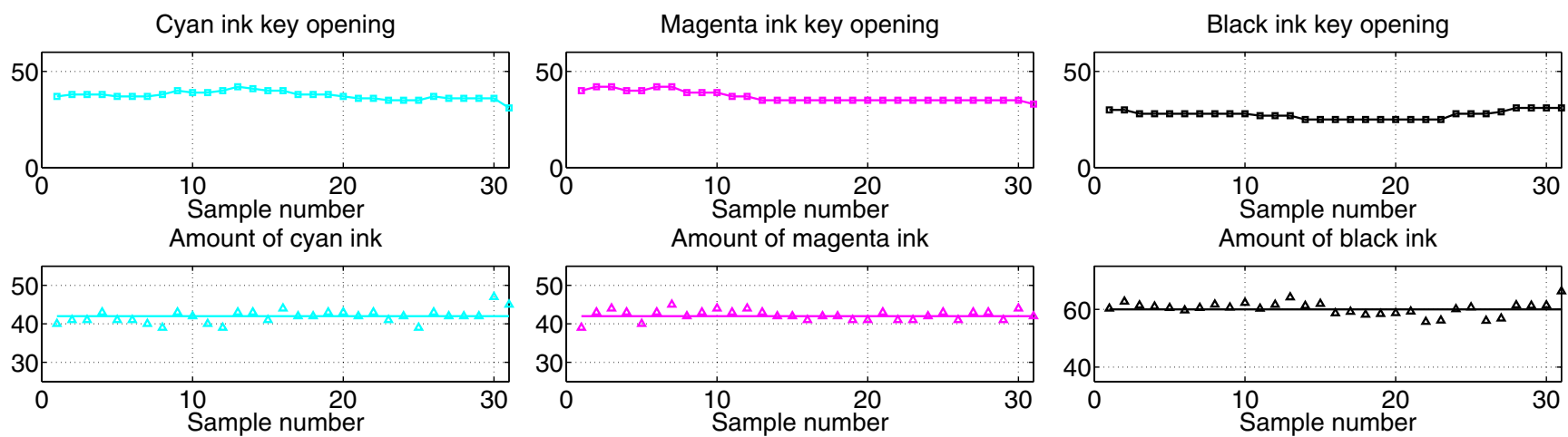

Fig. 16 The control signal (top) and the measured signal along with the desired (solid line) amount of ink (bottom) obtained from the RIFC 
Acknowledgements We gratefully acknowledge the financial support from the Knowledge Foundation Sweden, Holmen Paper, Stora Enso, and VTAB groups Sweden.

\section{References}

1. Sharma G, Trussell HJ (1997) Digital color imaging. IEEE Trans Image Process 6:901-932

2. Bralasubramanian R (1999) Optimization of the spectral Neugebauer model for printer characterization. J Electron Imaging $8: 156-166$

3. Pappas T (1997) Model-based halftoning of color images. IEEE Trans Image Process 6:1014-1024

4. Almutawa S, Moon YB (1999) The development of a connectionist expert system for compensation of color deviation in offset lithographic printing. Artif Intell Eng 13:427-434

5. Brown N, Jacksson M, Bamforth P (2004) Machine vision in conjunction with a knowledge-based system for semi-automatic control of a gravure printing process. In: Proceedings of the I MECH E part I journal of systems \& control engineering, vol 218. Professional Engineering Publishing, pp 583-593

6. Pope B, Sweeney J (2000) Performance of an online closed-loop color control system. In: TAGA 2000 proceedings, Technical Association of the Graphic Arts, Sewickley, PA, pp 417-431
7. Wyszecki G, Stiles WS (1982) Color science. Concepts and methods, quantitative data and formulae, 2nd edn. Wiley, New York

8. Verikas A, Malmqvist K, Bergman L (2000) Neural networks based colour measuring for process monitoring and control in multicoloured newspaper printing. Neural Comput Appl 9:227-242

9. Chen L, Narendra KS (2001) Nonlinear adaptive control using neural networks and multiple models. In: Proceedings of the American control conference, vol 6. IEEE, Chicago, Illinois, pp 4199-4203

10. Ravindranathan M, Leitch R (1999) Model switching in intelligent control systems. Artif Intell Eng 175-187

11. Yu W (2006) Multiple recurrent neural networks for stable adaptive control. Neurocomputing 70(1-3):430-444. DOI 10.1016/j.neurocom.2005.12.122

12. Englund C, Verikas A (2007) A SOM based data mining strategy for adaptive modelling of an offset lithographic printing process. Eng Appl Artif Intell 20:391-400

13. Englund C, Verikas A (2006) Ink flow control by multiple models in an offset lithographic printing process. Comp Ind Eng (submitted)

14. Englund C, Verikas A (2005) A SOM based model combination strategy. In: Wang J, Liao X, Yi Z (eds) Lecture notes in computer science, vol 3496, part 1. ISNN 2005, Chongqing, China, Springer, Berlin Heidelberg New York, pp 461-466

15. Herzallah R, Lowe D (2004) A mixture density network approach to modelling and exploiting uncertainty in nonlinear control problems. Eng Appl Artif Intell 17:145-158 